\title{
Hipertensão e obesidade em um grupo populacional no Nordeste do Brasil
}

\section{Hypertension and obesity in a population group from the Northeast of Brazil}

Maria Olganê Dantas SABRY1

Helena Alves de Carvalho SAMPAIO'

Marcelo Gurgel Carlos da SILVA²

RESUMO

O objetivo deste estudo foi identificar a prevalência de hipertensão arterial e obesidade, bem como sua inter-relação em funcionários de uma instituição de ensino superior da cidade de Fortaleza, Ceará. Foram entrevistados e avaliados, a partir de dados antropométricos e de pressão arterial, 317 funcionários. Para a aferição e classificação da pressão arterial, foram adotados os critérios do III Consenso Brasileiro de Hipertensão Arterial de 1998 e do Joint National Committee de 1997. Foi utilizado o Índice de Massa Corporal para a classificação nutricional, segundo critérios da Organização Mundial da Saúde de 1995. Encontrou-se uma prevalência de hipertensão arterial de $25,6 \%$ e de excesso de peso de 59,9\%, ambas significativamente mais prevalentes no sexo masculino $(p<0,05)$. A obesidade foi fator de risco para hipertensão arterial no grupo estudado ( $O R 7,53 ; I_{95} 3,08-18,92 ; p=0,00$ ). Os resultados encontrados enquadram a comunidade pesquisada como grupo alvo de ações de saúde destinadas ao controle das doenças crônicas.

Termos de indexação: obesidade, hipertensão, prevalência.

The aim of this study was to identify the prevalence of arterial hypertension and obesity, as well as their interrelationship, in 317 employees of a university in the city of Fortaleza, in state of Ceará. These employees were interviewed and anthropometric and arterial pressure data were collected. The parameters used to

\footnotetext{
1 Departamento de Ciências da Nutrição, Centro de Ciências da Saúde, Universidade Estadual do Ceará. Av. Paranjana 1700, Campus de Itaperi, 60740-400, Fortaleza, CE, Brasil. Correspondência para/Correspondence to: M.O.D. SABRY.

2 Departamento de Saúde Pública, Centro de Ciências da Saúde, Universidade Estadual do Ceará.
} 
140 | M.O.D. SABRY et al.

obtain and classify arterial pressure were the ones from the III Arterial Hypertension Brazilian Consensus (1998) and from the Joint National Committee (1997). The body mass index was used to determine the nutritional classification, according to criteria from the World Health Organization (1995). It was found a prevalence of arterial hypertension of $25.6 \%$ and of overweight of $59.9 \%$, both more prevalent in males $(p<0.05)$. The obesity was a risk factor for arterial hypertension in the evaluated group (OR $7.53 ; I C_{95}$ 3.08-18.92; $p=0.00)$. These results showed that the studied community needs health activities directed towards controlling chronic degenerative diseases.

Index terms: obesity, hypertension, prevalence.

\section{NTRODUÇÃ O}

As doenças cardiovasculares passaram a representar a primeira causa de morte em todo o mundo (Brasil..., 1993a). Nos últimos 100 anos, a melhora das condições de vida das pessoas, embora insuficiente, levou a um controle na mortalidade por doenças transmissíveis, resultando no envelhecimento da população. Passaram, então, a predominar as doenças crônicas, particularmente as cardiovasculares, alterando todo o perfil de morbi-mortalidade dos diferentes países desenvolvidos (Araújo, 1992).

Apesar de as causas da maioria das doenças cardiovasculares serem desconhecidas, alguns fatores aumentam a probabilidade de sua ocorrência, destacando-se: hábitos alimentares, obesidade, aumento dos triglicerídeos e colesterol séricos, elevação da pressão arterial, alcoolismo, diabetes mellitus, fumo, hereditariedade, estresse e insuficiente atividade física (Brasil..., 1988; Brasil..., 1993a).

Com relação às doenças cardiovasculares no Brasil, mais especificamente à hipertensão arterial, não se conhece a prevalência da mesma em âmbito nacional, pois estudos representativos dos habitantes dos locais onde foram realizados não permitem qualquer inferência para o país como um todo, por não retratarem os atributos da sociedade brasileira (Lessa, 1993). Mesmo assim, baseado na visão do conjunto das diversas prevalências determinadas no Brasil, o Ministério da Saúde (Brasil...,1993b) inferiu para o país em sua totalidade uma prevalência de aproximadamente $15 \pm 3 \%$ na população de 20 anos ou mais. Conforme verificou-se também, mais da metade dos indivíduos portadores de hipertensão arterial não sabem que o são, pois a doença pode desenvolver-se de forma assintomática (Brasil..., 1988; Brasil..., 1993b).

A gravidade do problema da hipertensão arterial no país pode também ser aferida ao se constatar a classificação desta doença como a segunda maior causa de hospitalização por doença circulatória (21,4\%), tendo representado 2,5\% de todas as internações efetuadas na rede contratada pelo Instituto Nacional de Assistência Médica e Previdência Social (INAMPS), no primeiro semestre de 1987 (Brasil...,1993b). Segundo dados do Ministério da Saúde, no período de 1980-1986 esta doença foi a primeira causa de invalidez permanente no Brasil, contribuindo com $63,0 \%$ do total de aposentadorias e com 44,5\% dos auxílios-doenças concedidos pelo INAMPS, dentro do grupo de doenças do aparelho circulatório (Brasil..., 1993b). A hipertensão arterial primária ou essencial constitui o verdadeiro problema de saúde pública (95,0\% dos casos), e a combinação de fatores genéticos, familiares e ambientais parece ser a principal responsável por esta condição (Brasil..., 1988).

Entre os fatores ambientais destaca-se a obesidade. De acordo com estudos epidemiológicos mundiais, a obesidade e a pressão arterial estão estreitamente associadas, porquanto a hipertensão é encontrada freqüentemente em 
pessoas obesas, bem como naquelas que ganham peso (Joint National..., 1997; Consenso Brasileiro..., 1998). No Brasil, dados de Piracicaba, SP, revelaram obesidade em $38 \%$ dos indivíduos hipertensos (Ayres, 1991). Confirmando esta associação, observa-se geralmente diminuição apreciável da pressão arterial provocada pela redução de peso, mesmo não sendo atingido o ideal (Joint National..., 1997).

O Consenso Brasileiro... (1998), considerando que a pressão arterial exibe uma relação direta com o ganho ponderal e o controle deste conduz à redução dos níveis pressóricos, recomenda a inclusão de todos os hipertensos com excesso ponderal em programas de redução de peso, de modo a alcançarem, idealmente, um Índice de Massa Corporal (IMC) inferior a $25 \mathrm{~kg} / \mathrm{m}^{2}$. Esta mesma proposição é adotada pelo Joint National Committee, embora com diferente IMC considerado de risco, que é maior ou igual a $27 \mathrm{~kg} / \mathrm{m}^{2}$ (Joint National..., 1988).

Assim, examinando os dados da literatura mundial, os quais associam a obesidade à hipertensão arterial, e levando-se em conta a lacuna no conhecimento da situação na cidade de Fortaleza, tanto no tocante à prevalência de hipertensão arterial quanto de obesidade, pretendeu-se com o presente estudo estimar estas prevalências em funcionários adultos de uma Instituição de Ensino Superior da referida cidade, bem como inter-relacioná-las. Uma limitação desta pesquisa é o fato de a mesma não ser necessariamente representativa da população adulta da cidade. No entanto, espera-se que ela venha a ser um alerta para esta problemática, gerando estudos posteriores de maior abrangência e representatividade.

\section{CASUISTICAE METODOS}

Esta pesquisa foi realizada na Universidade Estadual do Ceará (UECE), no Campus do Itaperi, em Fortaleza, e se propôs a obter dados antropométricos e de pressão arterial de todos os funcionários técnico-administrativos com regime de trabalho de 40 horas semanais.

Do total de funcionários cadastrados no Departamento de Recursos Humanos da Instituição, foram excluídos aqueles que se encontravam afastados e não retornariam por ocasião da coleta de informações, restando 389 funcionários efetivos e em atividade no momento do levantamento dos dados, realizado nos meses de agosto e setembro de 1998. Destes, 30 recusaram participação no estudo e 42 faltaram a alguma etapa programada, resultando na inclusão de 317 indivíduos no trabalho (81,5\% dos funcionários). O projeto da pesquisa foi aprovado pelo Comitê de Ética em Pesquisa da UECE e todos os participantes assinaram um termo de concordância de participação no estudo, após serem informados dos objetivos do mesmo. Inicialmente os funcionários responderam a um questionário contendo dados socioeconômicos, que permitiram a caracterização dos mesmos.

Para medir a pressão arterial foi usado esfigmomanômetro de mercúrio (Brasil..., 1988; Consenso Brasileiro..., 1998), marca Wanross, e estetoscópio clínico, marca BD. O manguito utilizado foi o de $14 \mathrm{~cm}$ de largura, preconizado pela Organização Mundial da Saúde (OMS), para permitir a inclusão também de indivíduos adultos obesos (Brasil..., 1993b). Para a determinação da pressão arterial foi treinado um único Auxiliar de Enfermagem, o qual aferiu a pressão de todos os funcionários, seguindo procedimentos rigorosamente normatizados. Utilizou-se método auscultatório indireto em duas ocasiões diferentes, com intervalo de $5 \pm 2$ dias. Os indivíduos foram previamente orientados a não fumar, beber café e se alimentar nos 30 minutos antecedentes à aferição da pressão arterial (Joint National..., 1997; Consenso Brasileiro..., 1998). O seguimento desta orientação foi confirmado no momento da obtenção das medidas. Para evitar variações acentuadas da pressão arterial, estas foram obtidas após cinco minutos de repouso e relaxamento. Para aferição os indivíduos ficaram sentados com o braço repousado sobre uma superfície firme e à 
altura do precórdio (Brasil..., 1988; Joint National..., 1997; Consenso Brasileiro..., 1998).

Na primeira ocasião a pressão arterial foi medida em ambos os braços, seguindo a recomendação de vários autores (Brasil..., 1988; Ayres, 1991; Consenso Brasileiro..., 1998). Realizaram-se duas aferições em cada braço com intervalo de um minuto, e estabeleceu-se a média de cada braço. Na segunda ocasião utilizou-se apenas o braço direito, efetuando-se duas medidas, com intervalo de um minuto, e estabelecendo-se a média. Foi registrado em cada medida o I ruído de Korotkoff como pressão sistólica e o V ruído como pressão diastólica (Brasil..., 1988; Brasil..., 1993b; Joint National..., 1997; Consenso Brasileiro..., 1998).

Foi considerado hipertenso o indivíduo que apresentou pressão arterial sistólica (PAS) maior ou igual a $140 \mathrm{mmHg}$ e/ou pressão arterial diastólica (PAD) maior ou igual a $90 \mathrm{mmHg}$ em duas verificações em dias diferentes, conforme critérios estabelecidos pelo Joint National Committee (Joint National..., 1997).

Por ocasião da primeira aferição da pressão arterial, foram levantados os dados antropométricos de peso e altura para determinação do IMC. Estas medidas foram obtidas segundo padronizações de Gouveia (1978), utilizando-se balança antropométrica Filizola, modelo 31, capacidade de $150 \mathrm{~kg}$ e graduação de $100 \mathrm{~g}$. Para a aferição da altura utilizou-se antropômetro contido na própria balança. O IMC foi determinado através da fórmula peso $(\mathrm{kg}) /$ altura $^{2}(\mathrm{~m})$ e foi classificado o estado nutricional segundo critérios preconizados pela World Health... (1995), ou seja, desnutrição grau 3 (IMC < 16,00), desnutrição grau 2 (IMC 16,00-16,99), desnutrição grau 1 (IMC 17,00-18,49), normal (IMC 18,50-24,99), sobrepeso (IMC 25,00-29,99), obesidade grau 1 (IMC 30,00-34,99), obesidade grau 2 (IMC $35,00-44,99)$ e obesidade grau 3 (IMC $\geq 45,00$ ).

A prevalência de hipertensão arterial foi avaliada segundo o sexo, utilizando-se o teste do $\chi^{2}$ para verificar a significância das diferenças encontradas e adotando-se intervalo de confiança de $95 \%\left(I C_{95}\right)$. A prevalência encontrada foi ajustada por sexo, considerando a distribuição da população total por idade, e foi também padronizada por sexo conforme a distribuição de peso da população total em estudo.

Para se definir a presença de associação entre hipertensão arterial e obesidade, foi calculada a odds ratio (OR), $(p<0,05)$, utilizando-se os pacotes estatísticos do Epi Info, versão 6.0 e do Stata 4.0. O ponto de corte para considerar obesidade como risco para hipertensão arterial foi IMC $>24,99$, uma vez que este é o valor máximo de normalidade segundo a World Health... (1995).

RESULTADOS

\section{Caracterização dos funcionários entrevistados}

Dos 317 funcionários pesquisados, 157 $(49,5 \%)$ eram do sexo masculino e 160 (50,5\%), do feminino. A idade média do sexo masculino foi de 43,2 anos, com variação de amplitude de 27 a 70 anos, e a do feminino foi de 40,9 anos, com limites de 27 a 64 anos. A idade média global do grupo estudado foi de 42 anos, com uma amplitude de 27 a 70 anos. Houve maior concentração de indivíduos nas faixas etárias de 30 a 39 anos - $135(42,6 \%)$ e 40 a 49 anos - 120 $(37,8 \%)$. Estes dois grupos comportaram 80,8\% do total de funcionários pesquisados.

A maioria dos funcionários (213 - 67,2\%) tinha escolaridade equivalente ao Ensino Médio completo ou acima deste nível, e 80 deles (25,3\%) tinham instrução maior ou igual ao Superior completo.

Quanto à renda média familiar, a mesma era elevada, ficando em torno de 12,7 salários mínimos (SM), com variação de 1,4 a 58,3 SM. Grande parte dos funcionários (139 - 43,8\%) possuía renda familiar acima de 10 salários mínimos. 


\section{Prevalência de hipertensão arterial}

Foram detectados 81 (25,6\%) indivíduos hipertensos, dos quais $57(70,4 \%)$ desconheciam esta condição. Dos 24 (29,6\%) que sabiam ser portadores de hipertensão, 9 referiram não adotar condutas de controle dos níveis pressóricos, enquanto os demais citaram uso de medicamentos isoladamente (4) ou associados à dieta (5), ao exercício físico (1) ou à dieta e exercício físico (1), além de uso exclusivo de dieta (4) (Tabela 1).

Observa-se que a prevalência de hipertensão arterial foi significativamente maior no sexo masculino - $63(40,1 \%)$ do que no feminino - $18(11,3 \%)\left(\chi^{2}=34,74, p=0,00\right)$. Este perfil foi mantido mesmo após ajuste por idade e sexo, conforme pode ser comprovado na referida tabela: $38,68 \%, \mathrm{IC}_{95}[31,06 \%-46,30 \%]$ para os homens e $11,33 \%, I C_{95}[6,42 \%-16,24 \%]$ para as mulheres.

A faixa etária que concentrou maior número de casos de hipertensão arterial, em ambos os sexos, foi a de 40-49 anos - 31 (38,2\%). Por outro lado, percebe-se uma maior prevalência da doença com o aumento da idade em ambos os sexos, à exceção da faixa etária de 20-29 anos, que mostrou uma das mais altas prevalências no sexo masculino.

\section{Hipertensão arterial e obesidade}

Constatou-se um percentual alto de indivíduos com IMC acima do normal, 190 $(59,9 \%)$, dos quais 136 (42,9\%) apresentaram sobrepeso e $54(17,0 \%)$ enquadraram-se na classificação de obesidade. Dos obesos, 45 $(14,2 \%)$ apresentavam obesidade grau I, 7 (2,2\%) grau II e $2(0,7 \%)$ grau III. Os funcionários com IMC normal compreenderam 123 (38,7\%), havendo ainda 4 (1,3\%) indivíduos com IMC compatível com desnutrição grau I. Particularizando por sexo, constatou-se que o sexo feminino exibiu um percentual de 54,4\% (87 funcionárias) com IMC elevado, sendo 14,4\% (23 funcionárias) compatíveis com obesidade. No sexo masculino encontrou-se um percentual mais elevado de IMC acima do normal - 65,7\% (103 funcionários), sendo 19,8\% (31 funcionários) compatíveis com obesidade. Houve diferença estatística entre os sexos considerando IMC acima do normal $\left(\chi^{2}=4,16 ; p=0,041\right)$, mas não ao se considerar apenas obesidade $\left(\chi^{2}=1,62 ; p=0,204\right)$ (Tabela 2).

Quanto aos hipertensos, a maioria encontrava-se com excesso de peso, sendo $46,9 \%$ (38 funcionários) com sobrepeso e 38,3\% (31 funcionários) com obesidade. Considerando os sexos, entre os homens havia 82,5\% (52 funcionários) com IMC acima do normal, dos quais

Tabela 1. Prevalência de hipertensão arterial (HA) nos funcionários estudados, segundo sexo e faixa etária.

\begin{tabular}{|c|c|c|c|c|c|c|}
\hline \multirow{2}{*}{ Sexo } & \multicolumn{3}{|c|}{ Masculino } & \multicolumn{3}{|c|}{ Feminino } \\
\hline & $\mathrm{F}$ & $\mathrm{H}$ & Prevalência de HA & $\mathrm{F}$ & $\mathrm{H}$ & Prevalência de HA \\
\hline Faixa Etária (anos) & $n$ & $\mathrm{n}$ & $\%$ & $n$ & $\mathrm{n}$ & $\%$ \\
\hline $20-29$ & 3 & 2 & 66,66 & 2 & - & - \\
\hline 30-39 & 59 & 19 & 32,20 & 76 & 5 & 6,57 \\
\hline $40-49$ & 59 & 24 & 40,67 & 61 & 7 & 11,47 \\
\hline $50-59$ & 24 & 10 & 41,66 & 20 & 6 & 30,00 \\
\hline $60-69$ & 11 & 7 & 63,63 & 1 & - & - \\
\hline $70 e+$ & 1 & 1 & 100,00 & - & - & - \\
\hline Total & 157 & 63 & 40,13 & 160 & 18 & 11,25 \\
\hline $\begin{array}{l}\text { Prevalência ajustada } \\
\text { poridadee sexo }\end{array}$ & - & - & 38,68 & - & - & 11,33 \\
\hline
\end{tabular}

Nota: $\mathrm{F}=$ funcionários; $\mathrm{H}=$ hipertensos 
31,7\% (20 funcionários) exibiam obesidade. Entre as mulheres, encontrou-se 94,4\% (17 funcionárias) com IMC elevado, dos quais $61,1 \%$ (11 funcionárias) com obesidade. Não houve diferença estatística entre os sexos, quer considerando IMC acima do normal $\left(\chi^{2}=0,12\right.$; $p=0,727)$, quer considerando apenas obesidade $\left(\chi^{2}=2,05 ; p=0,152\right)$.

A prevalência de hipertensão arterial foi maior entre os obesos - 57,4\%, sendo 51,1\% em obesos grau I, 100,0\% em obesos grau II e 50,0\% em obesos grau III. Quanto aos indivíduos com sobrepeso, a prevalência foi de $27,9 \%$, enquanto nos normais foi de apenas 9,8\%. Este perfil foi mantido mesmo após ajuste por peso e sexo (Tabela 2): 38,21\%, IC ${ }_{95}[30,61 \%$ - 45,81\%] para os homens e $12,62 \%, I C_{95}[7,48 \%-17,76 \%]$ para as mulheres.

O IMC elevado emergiu como fator de risco com uma odds ratio de 7,53 , intervalo de confiança de 3,08-18,92 e $p=0,00$.

A caracterização do grupo mostrou não terem sido expressivas entre os entrevistados a baixa escolaridade e baixa renda, citadas na literatura como fatores de risco para a hipertensão arterial. Mesmo assim foi realizado teste estatístico, confirmando-se que tais variáveis realmente não foram fatores de risco nos indivíduos estudados (escolaridade: OR 1,28; IC 0,61-2,69; p 0,59; renda: OR 0,83; IC $0,39-1,75$; p 0,72).

\section{DISCUSSÃO E CONCLUSÃO}

A caracterização apresentada foi realizada com o propósito de comparar o grupo entrevistado com os habitantes de Fortaleza. A distribuição entre os sexos não diferiu significativamente daquela da região metropolitana da cidade (Instituto Brasileiro..., 1998), onde há 47,3\% de homens e $52,7 \%$ de mulheres $\left(\chi^{2}=0,64 ; p=0,42\right)$. Já as faixas etárias mais freqüentes entre os entrevistados diferem significativamente das encontradas na população adulta da mesma localidade (Instituto Brasileiro..., 1998), onde há 27\% de indivíduos de 30-39 anos e 10\% de 40-49 anos (respectivamente $\chi^{2}=19,92 ; p=0,00$ e $\left.\chi^{2}=51,72 ; p=0,00\right)$.

O grupo estudado é ainda privilegiado em relação à realidade do Estado do Ceará e/ou cidade de Fortaleza no tocante aos aspectos de escolaridade e renda. Dados disponíveis do Instituto Brasileiro... (1998) referentes à região metropolitana do município apontam apenas 4,0\% dos indivíduos acima de 10 anos de idade com escolaridade $\geq$ ao ensino superior (15 ou mais anos de estudo), enquanto na presente pesquisa encontrou-se $25,3 \%\left(\chi^{2}=285,07 ; p=0,00\right)$. Em relação à renda familiar, os dados do Instituto Brasileiro... (1998) indicam que $84,7 \%$ dos habitantes recebem até 10 salários mínimos, enquanto $56,2 \%$ dos entrevistados neste estudo se concentraram nesta faixa $\left(\chi^{2}=229,71\right.$; $p=0,00)$.

Tabela 2. Prevalência de hipertensão arterial nos funcionários estudados, segundo sexo e diagnóstico nutricional.

\begin{tabular}{|c|c|c|c|c|c|c|}
\hline \multirow{2}{*}{ Sexo } & \multicolumn{3}{|c|}{ Masculino } & \multicolumn{3}{|c|}{ Feminino } \\
\hline & $\mathrm{F}$ & $\mathrm{H}$ & Prevalência de HA & $\mathrm{F}$ & $\mathrm{H}$ & Prevalência de HA \\
\hline Diagnóstico nutricional & $\mathrm{n}$ & $n$ & $\%$ & $\mathrm{n}$ & $\mathrm{n}$ & $\%$ \\
\hline Normal & 50 & 11 & 22,00 & 73 & 1 & 1,36 \\
\hline Sobrepeso & 72 & 32 & 44,44 & 64 & 6 & 9,37 \\
\hline Obesidadel & 27 & 16 & 59,25 & 18 & 7 & 38,88 \\
\hline Obesidade॥l & 4 & 4 & 100,00 & 3 & 3 & 100,00 \\
\hline Obesidade III & - & - & - & 2 & 1 & 50,00 \\
\hline Desnutriçãol & 4 & - & - & - & - & - \\
\hline Total & 157 & 63 & 40,13 & 160 & 18 & 11,25 \\
\hline $\begin{array}{l}\text { Prevalência ajustada por } \\
\text { sexo e diagnóstico } \\
\text { nutricional }\end{array}$ & - & - & 38,21 & - & - & 12,62 \\
\hline
\end{tabular}

Nota: $\mathrm{F}=$ funcionários; $\mathrm{H}=$ hipertensos 
A prevalência de hipertensão arterial, na faixa etária de 20-70 anos, encontrada nos funcionários da UECE foi de 25,6\%, de acordo com os critérios do Joint National Committee (Joint National..., 1997). Esta prevalência está acima da média nacional descrita, a qual corresponde a $15 \pm 3 \%$ da população de 20 anos ou mais de idade (Brasil..., 1993b). A referida estimativa da prevalência nacional foi realizada considerando principalmente os critérios da World Health... (1978)(160/95 mmHg), mas no Programa Nacional de Educação e Controle da Hipertensão Arterial (Brasil...,1988) é comentado que, se fosse utilizado o ponto de corte adotado pelo Joint National Committee (Joint National..., 1988), tal prevalência dobraria (ou seja, chegaria a 30,0\%). Nesta perspectiva pode-se inferir que os dados encontrados na UECE estão dentro da faixa nacional descrita. Não se pode desconsiderar, contudo, a utilização de diferentes critérios de diagnóstico na maioria dos estudos de prevalência realizados no Brasil de forma a não saber qual seria esta cifra nacional caso fossem empregados os valores de corte adotados na presente pesquisa.

Enfocando a variável sexo na prevalência da hipertensão arterial, uma publicação do Ministério da Saúde (Brasil..., 1993b) cita ser a mesma analisada em conjunto com a variável idade e refere ser tal prevalência maior em homens abaixo de 40 anos e em mulheres acima desta idade. Já Ayres (1991) não menciona sexo, mas apenas destaca o aumento da ocorrência da doença com a idade. Ribeiro \& Ribeiro (1986), na cidade de São Paulo, estudando 5 mil indivíduos, encontraram prevalência maior no sexo masculino quando acima de 44 anos, mas ela aumentou com a idade em ambos os sexos. No presente estudo, excetuando-se a faixa etária de 20-29 anos entre os homens, a prevalência de hipertensão arterial também aumentou com a idade em ambos os sexos, contrariando de certa forma as análises realizadas na publicação do Ministério da Saúde (Brasil..., 1993b).

A prevalência encontrada entre os funcionários da UECE não pode ser comparada com a realidade da cidade de Fortaleza ou do Estado do Ceará, pois não há estudos prévios conduzidos que tenham adotado a metodologia da presente pesquisa.

O encontro de $70,4 \%$ de desconhecimento da hipertensão por parte dos entrevistados está em consonância com citações de outros estudos nacionais (Brasil..., 1988; Brasil..., 1993b).

Constatou-se a presença de algum grau de excesso de peso em cerca de $60,0 \%$ dos funcionários pesquisados. Este percentual é maior do que os 32,0\% revelados pela Pesquisa Nacional sobre Saúde e Nutrição (PNSN), realizada em 1989 (Instituto Nacional..., 1991), para a população brasileira, utilizando o mesmo ponto de corte adotado neste estudo. A prevalência encontrada na PNSN (Brasil..., 1991) assemelhou-se à encontrada por Ayres (1991) em Piracicaba (38,0\%), embora este autor não tenha referido o critério para diagnóstico de obesidade adotado em seu estudo, bem como assemelhou-se à encontrada por Cervato et al. (1997) em Cotia, São Paulo (38,0\%), os quais utilizaram critérios semelhantes ao presente estudo para excesso de peso (IMC > 25,0). A PNSN (Instituto Nacional..., 1991) revelou também que no nosso meio o sobrepeso e a obesidade afetam proporcionalmente mais mulheres do que homens, à semelhança de outros países. Particularmente no Nordeste urbano, a referida pesquisa apontou prevalências de obesidade de 3,3\% entre os homens e 8,9\% entre as mulheres. Em levantamento junto às mulheres brasileiras, encontrou-se uma prevalência de 9,7\% de obesas e $25,1 \%$ de sobrepeso (Pesquisa Nacional..., 1997). Monteiro et al. (1995) analisando dados do IBGE referentes aos períodos de 1974/75 e 1989, encontraram prevalência crescente de obesidade, respectivamente 5,7 e 9,6\%. Nestes levantamentos o critério adotado para definir obesidade foi $I M C \geq 30 \mathrm{Kg} / \mathrm{m}^{2}$. Em relação ao sexo, estes autores verificaram prevalência maior no sexo feminino, nos dois períodos: em 1974/75, 8,2\% das mulheres e 3,1\% dos homens mostravam-se obesos; em 1989 houve um 
aumento para $13,3 \%$ entre as mulheres e 5,9\% entre os homens.

Entre os funcionários da UECE observou-se $9,8 \%$ de obesidade no sexo masculino e 7,3\% no feminino. Considerando tanto obesidade como sobrepeso, no grupo estudado verificou-se também uma prevalência maior no sexo masculino - 103 $(65,7 \%)$ do que no feminino - 87 (54,4\%). Merece investigação quais fatores podem estar contribuindo para estes resultados no grupo estudado. Pode-se supor o consumo alimentar ou a atividade física, mas, infelizmente, mais uma vez não há dados disponíveis a respeito que permitam discussão comparativa.

A prevalência de hipertensão arterial foi maior nos indivíduos com obesidade grau II - 100,0\% e foi decrescendo de acordo com a diminuição do excesso de peso, sendo apenas de 9,8\% nos indivíduos dentro da faixa de peso ideal. Realmente, analisando-se a Tabela 2, observa-se que, dos 81 hipertensos, 69 (85,2\%) exibiam sobrepeso ou obesidade. Ainda na mesma tabela, do total de 317 funcionários, havia 190 pessoas com sobrepeso e obesidade e, excluindo-se destes os 69 hipertensos, restam $121(51,3 \%)$ normotensos com IMC acima do normal.

Os dados obtidos neste estudo apontaram, portanto, a associação de hipertensão arterial ao excesso de peso, reforçando achados da literatura que demonstram o papel do excesso de peso no risco de hipertensão arterial (Joint National..., 1997).

Em suma, a prevalência de hipertensão arterial encontrada nos funcionários da UECE enquadra a comunidade pesquisada como grupo alvo de ações de saúde destinadas ao controle das doenças crônico-degenerativas. A presença de excesso de peso entre os indivíduos entrevistados, com proporção ainda maior entre os hipertensos, destaca também a necessidade de programas neste sentido. É recomendável investigar variáveis genéticas, bem como ambientais, com ênfase no comportamento alimentar deste grupo, a fim de se detectar outras diferentes inter-relações que melhor permitam direcionar ações de saúde necessárias.

\section{REFERENCIAS BIBLIOGRÁFICAS}

ARAÚjO, J.D. Polarização epidemiológica no Brasil. Informe Epidemiológico do SUS, Brasília, v.1, n.2, p.5-16, 1992.

AYRES, J.E.M. Prevalência da hipertensão arterial na cidade de Piracicaba. Arquivos Brasileiros de Cardiologia, São Paulo, v.57, n.1, p.33-36, 1991.

BRASIL. Ministério da Saúde. Secretaria de Assistência à Saúde. Departamento de Programas de Saúde. Coordenação de Doenças Cardiovasculares. Doenças cardiovasculares no Brasil - SUS. Brasília, 1993a. 36p.

BRASIL. Ministério da Saúde. Secretaria de Assistência à Saúde. Departamento de Programas de Saúde. Coordenação de Doenças Cardiovasculares. Controle da hipertensão arterial: uma proposta de integração ensino-serviço. Rio de Janeiro: CDVS/NUTES, 1993b. 233p.

BRASIL. Ministério da Saúde. Secretaria Nacional de Doenças Crônicas-Degenerativas. Programa Nacional de Educação e Controle da Hipertensão Arterial. Normas técnicas para o Programa Nacional da Hipertensão Arterial (PNECHA). Brasília : Centro de Documentação do Ministério da Saúde, 1988. $88 p$.

CERVATO, A.M., MAZZILLI, R.N., MARTINS, I.S., MARUCCI, M.F.N. Dieta habitual e fatores de risco para doenças cardiovasculares. Revista de Saúde Pública, São Paulo, v.31, p.227-235, 1997.

CONSENSO BRASILEIRO DE HIPERTENSÃO ARTERIAL, III - 1998. Disponível em: $<w w w . s b n$.org.br/consiiiO.htm>. Acesso em: 1 maio 1999.

GOUVEIA, E.L.C. Diagnóstico do estado nutricional da população. In: CHAVES, N. Nutrição básica e aplicada. Rio de Janeiro : Guanabara Koogan, 1978. p.245-271.

INSTITUTO BRASILEIRO DE GEOGRAFIA E ESTATÍSTICA. Pesquisa nacional por amostra de domicílios - 1997. Rio de Janeiro : IBGE, 1998. p.68-119.

INSTITUTO NACIONAL DE ALIMENTAÇÃO E NUTRIÇÃO. Pesquisa Nacional sobre Saúde e 
Nutrição (PNSN) 1989: condições nutricionais da população brasileira: adultos e idosos. Brasília : Ministério da Saúde, 1991. 39p.

JOINT NATIONAL COMMITTEE. The 1988 report of the Joint National Committee on detection, evaluation, and treatment of high blood pressure. Archives of Internal Medicine, Chicago, v.148, p.1023-1038, 1988.

JOINT NATIONAL COMMITTEE. The sixth report of the Joint National Committee on prevention, detection, evaluation, and treatment of high blood pressure. [s.l.:s.n.], 1997. 70p. (NIH Publication, n.98-4080).

LESSA, I. Estudos brasileiros sobre a epidemiologia da hipertensão arterial: análise crítica dos estudos de prevalência. Informe Epidemiológico do SUS, Brasília, v.2, n.3, p.59-75, 1993.

MONTEIRO, C.A., MONDINI, L., SOUZA, A.L.M., POPKIN, B.M. Da desnutrição para a obesidade: a transição nutricional no Brasil. In: MONTEIRO, C.A. (Org.). Velhos e novos males de saúde no Brasil. São Paulo : Hucitec, 1995. p.247-255.

PESQUISA nacional sobre demografia e saúde. Rio de Janeiro : Benfam, 1997. 180p.

RIBEIRO, A.B., RIBEIRO, M.B. Epidemiological and demographic considerations. Hypertension in underdeveloped countries. Drugs, New York, v.31, p.23-28, 1986.

WORLD HEALTH ORGANIZATION. Expert Committee on Arterial Hypertension. Geneva, 1978. 58p. (Technical Report Series, 628).

WORLD HEALTH ORGANIZATION. Expert Committee on Physical Status: the use and interpretation of anthropometry, Geneva, 1995. 452p.

Recebido para publicação em 4 de outubro de 2000 e aceito em 30 de março de 2001. 